\title{
Lung Cancer pMX TNM Finding v6
}

National Cancer Institute

\section{Source}

National Cancer Institute. Lung Cancer pMX TNM Finding v6. NCI Thesaurus. Code C60977.

Lung cancer in which distant metastasis cannot be assessed. (from AJCC 6th Ed.) 